\title{
Simulation of Berkovich nanoindentation experiments on thin films using finite element method
}

\author{
M. Lichinchi ", C. Lenardi it* , J. Haupt ", R. Vitali"

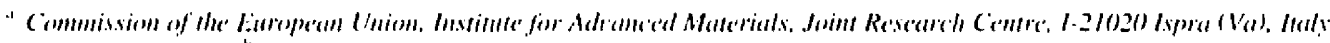

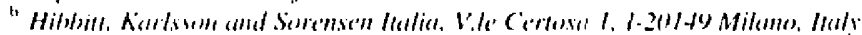

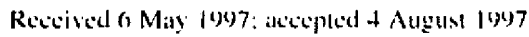

\begin{abstract}
The Finite element technique is applied for studying the very complex stre!s--strain field of thin halrd coatings subjected to a nancindentation process. Berkovich indentation experiments were simulated with the ABAQUS finite element software package. The investigated system was titanium nitride on high speed sleel as an example of a hard film on a softer substrate. The numerical analysis allowed the plastic deformation history during inclentation to be followed. In particular. it was possible to correlale lle onset of plastic deformation in the substrate with the shape of the loading curve. The system wals simulated by all axisymmetric mudel in which the conical indenter has the same contact area as the Berkovich indenter. A six-fold symmetric three-dimensional model was also defined lor testing the suitability of the previous model. The indenter was modeled either ats a rigid surfiace or as a deformable diamond tip. Comparison between the experimental data and ntmerical results demomstrated that the finite element approatch is capable of teproducing the loading--unloading behavior of a nanoindentation test. The film hardness of TiN/HSS specimens was numerically calculated for different indentation depths. It was shown that the presence of the substralle alfected the hardness measurenent lor eleltive indentation depths greater than about $15 \%$ of the film thickness. al 1908 Elsevier Scicince $S . A$.
\end{abstract}

Kevwords: Jïnite element methed: Berkovich nanoindentaion proces: TiN/HSS

\section{Introduction}

The mechanical surfice properties of many materials can be improved by depositing uppropriate coatings. in particular with respect to wear resistance. friction coefficient. hardness, etc. Local deformations of coated surfaces produced by tribological interactions can result in substantial damage. Thus, it is important to quantify the resistance of the material to such damage. For this reason the evaluattion of the hardness as obtained from indentation measurements is of fundamental importance. Elastic modulus and hardness are the two properties that are more frequently measured by the load and depth serising indentation lechnique. Theoretical analysis of general indentation problems has received great attention from many investigators. A few models are hased on the classic slip line theory for rigid-plastic solids [1]. A new approach was introduced by Tabor [2] who analyzed the plastically deformed zone as the expansion of a spherical cavity in an elastic-plastic

\footnotetext{
Comrespomeling atuthor.
}

material having an internal hydrostatic pressure. Johnson [3] added to this approach the effect of the tip geometry. However, all the proposed models cannol describe the very complex streis and stratin lie!d produced during the indentation process. Since the induced plastic deformation depends on the path through which eatch indentation step is reached, it should be very useful to be able to analyze the deformation history.

Some investigators have studied the indentation process using the numerical approatch of finite element method. Cai and Bangert [4] studied with this method the extension of the plastic cuformed zone in relation to the ratio of the penetration depth to the film thickness $d / t$, and determined the effect of the substrate on the measured hardness. In the case of soft films on hard substrilles they found thall this effect was still negligible up to $d / 1 \times 100 \approx 30 \%$. On the contrary. for hard films on softer substrates, the plastic deformation extended eatsily into the substrate. and the measured hardness was modified even for $d / 1 \times 100=$ $10 \%$. Sun et al. [5] employed an axisymmetric FEM model 10 simulate a hard coatling on a softer substritte. A conical 
indenter having the same projected area-depth function as the Berkovich indenter was assumed. The nanoindentation process for various coating-to-substrate yield strength ratio was analyzed. Moreover, the effect of the indenter tip radius was taken into account. Sun et al. [5] determined that an increase of the yield strength ratio or of the tip radius reduced the critical ratio $(d / t)_{\text {sri }}$ for which the hardness measurements were not affected significantly by the substrate. A three-dimensional FEM analysis was performed by Wang and Bangert [6] who investigated the behavior of coated systems during a Vickers indentation process. The results showed that the first part of the loading curves closely followed the behavior of the bulk coating and from $d / 1 \times 100=10 \%$ the eflect of the substrate became evident. A first example of comparison between FEM analysis and experimental results was presented by Bhatlacharya and Nix [7] in which they simulated a sub-micrometer indentation lest. They verified that the finite element method could be an effective tool for simulating hardness measurements.

In our work, we used the finite alement method to simulate the nanoindentation process. At first we compared the experimental load-depth response of titanium nitride thin films on high speed steel substrates with numerical results. Successively, we studied the effect of the substrate on the hardness measurements. Due to the complexity of the phenomena involved in the indentation process, we used the FE program ABAQUS [8] which allows eflective modeling of non-linear problems such as the materials properties. the contact between two hodies and the large deformations of the matterial under the indenter.

\section{Nanoindentation measurenents}

The nanoindentation technique allows Young's modulus and the hardness of thin films to be evaluated. In this section, we describe how a nanoindentation mensurement is performed and how the experimental results are elaborated for determining the mechanical properties. Nanoindentation measurements are pefformed uncior a constant load rate control. When the tinal depth is reached. a constant load is maintained to allow for possible creep before the unloading with a constant displacemen' rate is performe d. In Fig. I. a schematic draw of an indsstation load-disflacement curve without the constant load segment is hown.

As reported by Otiver and Pharr [9] and Brotzen [10] the effects of a non-rigid indenter on the load displacement behavion can be taken into account by defining an affective modulus $E_{\mathrm{r}}$

$\frac{1}{E_{\mathrm{r}}}=\frac{1-\nu^{2}}{E}+\frac{1-\nu_{1}^{2}}{E_{1}}$.

where $E$ is the elastic modulus and $y$ is the Poisson's ratio of the specimen. and $E$, and $\nu$, are the same parameters for the indenter. $E_{\mathrm{r}}$ is evaluated from the nanoindentation measurements according to the following equation

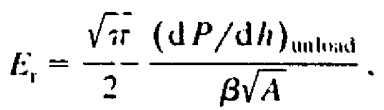

where $\Rightarrow$ is the indenter load. $h$ the penetration depth and $(\mathrm{d} P / \mathrm{d} / \mathrm{l})_{\text {unluat }}$ is the slope of the unloading curve evaluated at the position of maximum load. $A$ i. the contact

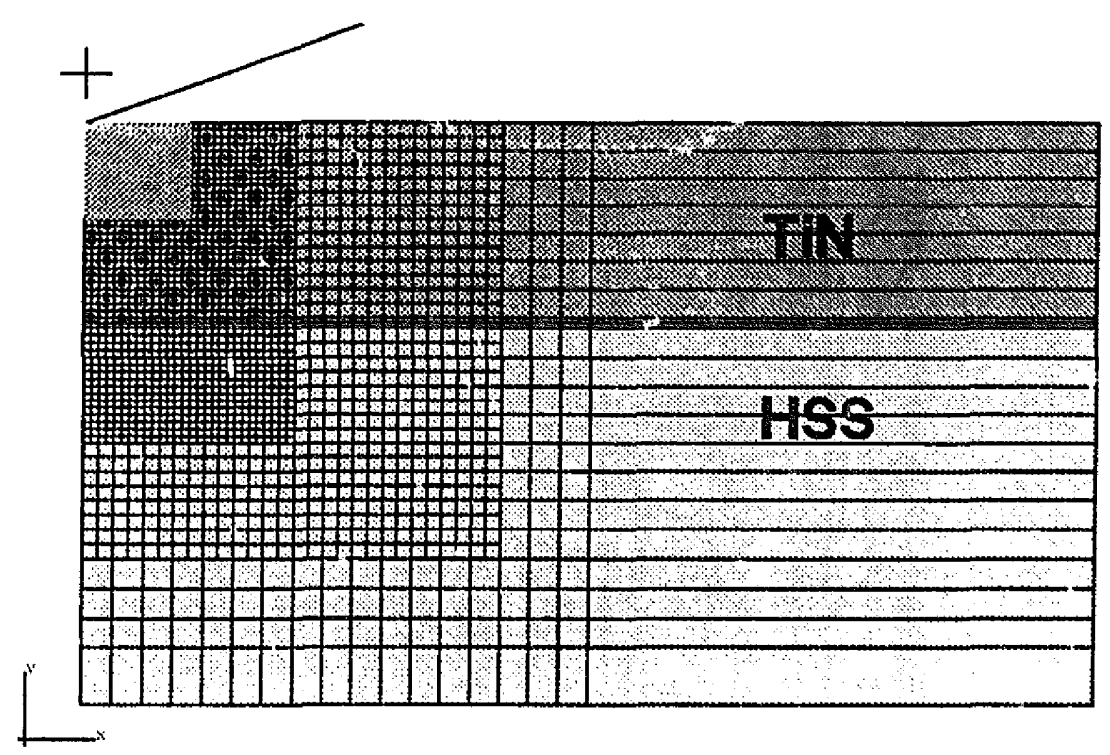

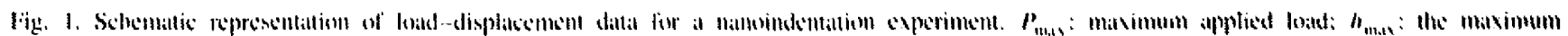

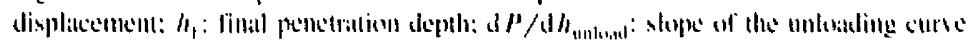


area and $\beta$ is a dimerisionless parameter related to the geometry of the indenter. In the case of a conical indenter $\beta=1$.

For evaluating the elastic modulus $E_{1}$. the slope $(\mathrm{d} P / \mathrm{d} h)_{\text {unloul }}$ and the contact area $A$ should be determined precisely. A least mean square fit $1090 \%$ of the unloading curve is made according to the hypothesis that the unloading data will be better expressed by a power law

$P=P_{\max }\left(\frac{h-h_{1}}{h_{\operatorname{mix}}-h_{i}}\right)^{\prime \prime}$.

$P_{\text {mixx }}$. $h_{\text {mixix }}$ are the maximum load and the maximum displacement respectively. while the final penetration depth $h_{i}$ and the exponent $m$ are the parameters to be litted. Eq. (3) is than analytically differentiated for determining the slope $(\mathrm{d} P / \mathrm{d} h)_{\text {unlowid }}$.

The contact area $A$ is given as a function of the contact depth $h_{\mathfrak{i}}$. The contact depth at maximum load is then

$h_{\mathrm{c}}=h_{\text {man }}-\epsilon\left(h_{\text {min }}-h_{1}\right)$

where $\epsilon=0.72$ for a conical indenter as given by Sneddon [11]. The expression for the contact area for a Berkovich indenter is usually approximated by the formula

$A\left(h_{\mathrm{c}}\right)=24.5 h_{\mathrm{c}}^{2}+\sum_{i=0}^{7} C_{1} h_{\mathrm{c}}^{1 / 2}$

where the first term gives the contact area of an ideal indenter whereas the terms preselli in the sum tuhe inn account geometrical deviations due to the tip rounding.

The Young's modulus $E$ is obtained from Eqs. (1) and (2). and the hardness $H$ is given by the well-known relation

$H=\frac{P_{\operatorname{mix}}}{A}$.

\section{FEM modeling}

In the present work, we have studied the nanoindentition process of a TiN coating grown on a HSS substrate. The nanoindentation measurements have been performed with the depth sensing Nanoindenter II of Nano Instruments equipped with a Berkovich diamond tip. We have simulated the process using an axisymmetric model which allows mesh definition with a restricted number of degrees of freedom. However. we have verified if this modeling is appropriate by performing an analysis with a threc-dimensional model.

\section{I. Axisymmetric model}

In order to define an axisymmetric model, we have used an equivalent conical indenter with a semi-apical angle of $y=70^{\circ} 30^{\prime}$. This system has geometric and loading symmetry around the axis of the indenter. The specimen is modeled with 26.35 4-node axisymmetric reduced integration elements (CAX4R element type [i] ). as shown in Fig. 2. The indentation region is very small with respect to the size of the sample. A high mesh retinement is used for modeling the large deformation area. In the outer zones multi-point constraints (MPCs type linear) atre introduced for interfacing one element with two adjacent elernents to reduce the total number of degrees of freedom. Finally semi-infinite elements (CINAX4 element type [8]) resemble the far field domain in the radial direction. The elements at the interlayer between the film and the substrate are reduced in height to be able to detect more accurately the behavior of that zone. The imposed geometrical boundary conditions are: the nodes along the axis of rotation can move only along such an axis, i.e., the y axis, and all the nodes on the bottom of the mesh are fixed. i.e. the displacements along $x$ and $y$ direction. are constrained.

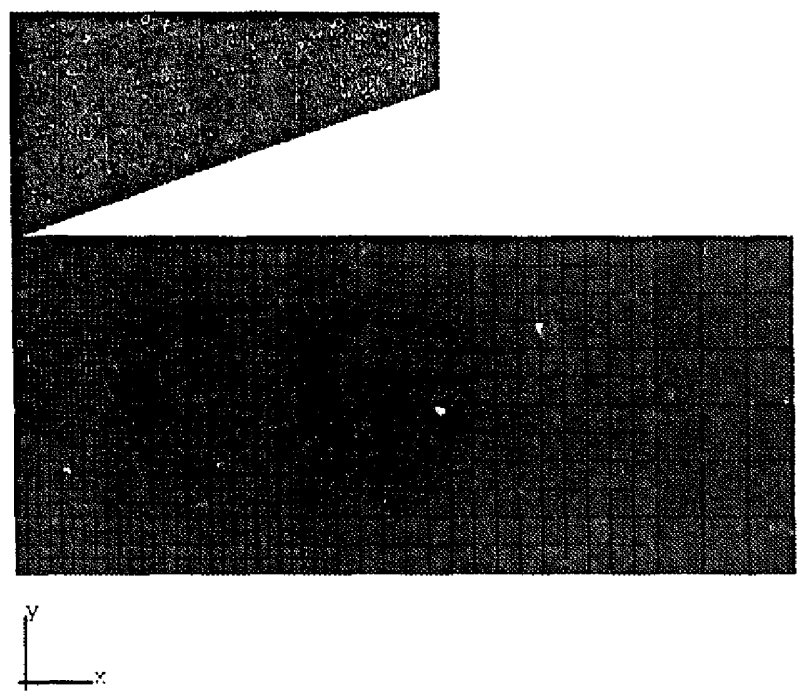

ligh. 2. Mesh of the whole satmple with the indenter modeled as at movable rigid surlices. 


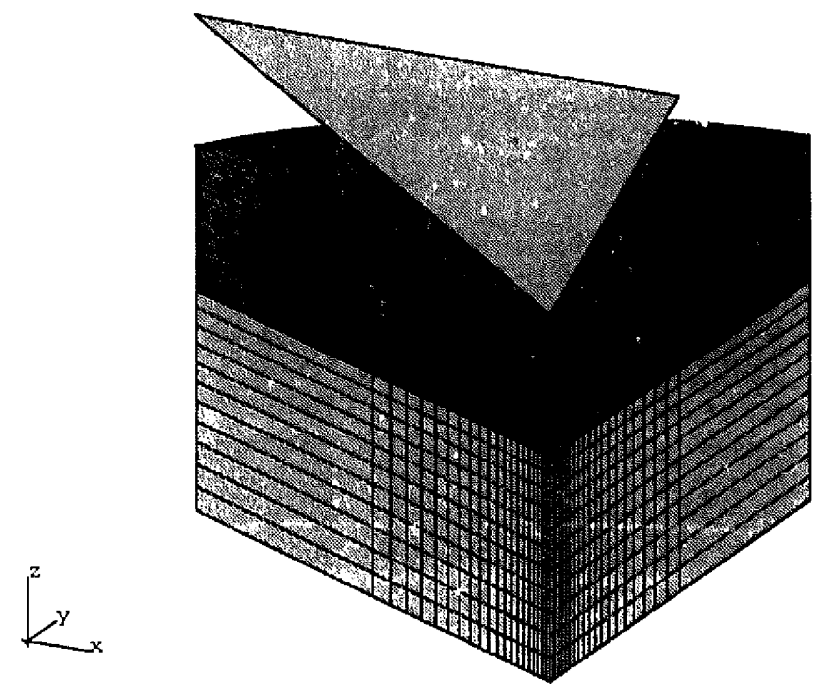

Fig. 3. Detatil of the indentation region with the indenter modeled as an clastic-plastic tip.

An important feature of ABAQUS is its capability to model the contact between two bodies as a sliding through contacting surfaces, which are in our case the indenter surface and the specimen surface. From the initial geomeIry the nodal gaps between the surfaces are defined. During the analysis, the program controls the variation of the nodal gaps. In particular whenever the distance between the indenter and the specimen becomes zero-gap closure - the contact takes place and an external reaction force is exerted on the nodes involved in the contact. The contact constraint is enforced by the definition of the "master" and the 'slave' surfaces: only the master surface can penetrate into the slave surlice and the contact direction is always taken as being normal to the master surface. We have chosen the indenter surfice as the master surface due to the larger stiffness of the indenter with respect to the specimen. In Fig. 2 the indenter is represented by a rigid movable surface. Since the coating is very hard, we have also modeled the tip with axisymmetric elements for taking into account the stilfness of the indenter. This mesh is shown in Fig. 3.

The friction coefficient between the tip and the specimen surface is assumed to be zero, becaluse in the case of a hard coating on a softer substrate, like TiN/HSS system. the friction has a negligible elfect on the nanoindentation process. '

Coating and substrate are assumed to be initially stress free and in perfect contact during the indentation process.

Both the TiN coating and the HSS substrate are assumed to be homogeneous and isotropic and having a perfect elastic-plastic behavior.

The indentation procedure is simulated by two subse-

\footnotetext{
'K. Matsudia. Private c(ommunnication.
}

quent steps: loading and unloading. During loading. the rigid surface or the modeled tip moves along the ?' direction (see Figs. 2 and 3) and penetrates the specimen up to the maximum depth; during unloading, the tip returns to the initial position. At each depth increment, the program makes many iterations according to a specified convergence rate to reach an equilibrate and congruent configuration.

Purely elastic deformation takes place only during the beginning of the indentation process. The Mises yield criterion is applied for determining the occurrence of the plastic deformation. The equivalent Mises stress is given by the expression

$\sigma_{\mathrm{Mi} \text { L }}=\sqrt{\frac{\left(\sigma_{1}-\sigma_{2}\right)^{2}+\left(\sigma_{2}-\sigma_{3}\right)^{2}+\left(\sigma_{3}-\sigma_{1}\right)^{2}}{2}}$.

where $\sigma_{1}, \sigma_{2}$ and $\sigma_{3}$ are the three principal stresses.

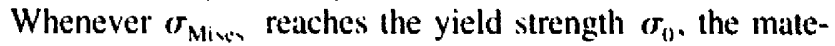
rial begins to deform plastically.

\subsection{Three-dimensional model}

The Berkovich indenter is a triangular-base pyramid having a six-fold symmetry. The load is applied along the axis of the indenter, thus the load symmetry is the same as the geometric one. For these considerations. a thiee-dimensional model is defined only by one sixth of the entire system. The specimen is modeled with 6925 8-node reduced integration elements (C3D8R element type [8]) and with semi-infinite elements (CIN3D8 element type [8]). The three-dimensional mesh is reported in Fig. 4. The large deformation area is modeled with elements smaller than these in the outer zone. We used a courser mesh with respect to the axisymmetric model in order to limit the degrees of treedom. Due to the fact that in the axisymmetric analysis the results for a rigid and for a deformable indenter are very similar. as described below. we have modeled the indenter with a rigid movable surface.

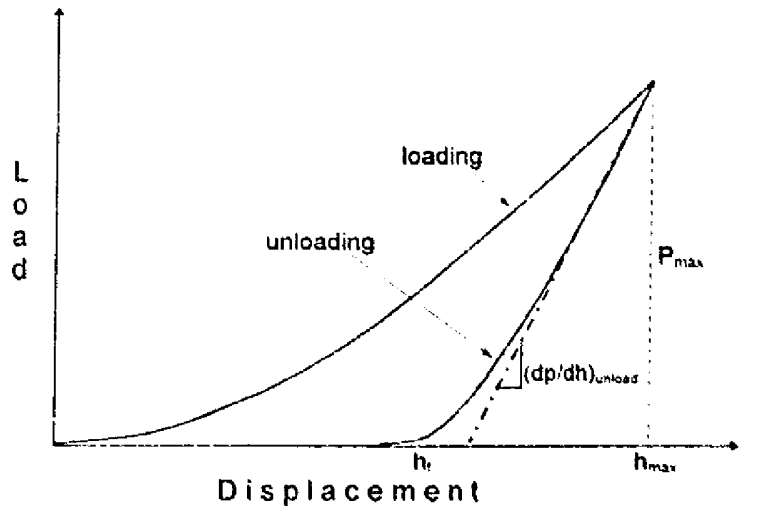

Fig. + . There-dimensional mentel. The Berkowich indenter has a six-liold symmety and the lonal is applied atong the axis al the indenter. The mest is detined only lor one-sixth of the șvem. 
Titble I

Matcrial properties of TiN, HSS and dianmond used in the lintite clement simulations

\begin{tabular}{|c|c|c|c|c|c|}
\hline & 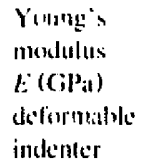 & $\begin{array}{l}\text { Young's modulus } t: \\
\text { (cil'il) righid indenter }\end{array}$ & $\begin{array}{l}\text { Poissonl"s } \\
\text { ratio } 1 .\end{array}$ & $\begin{array}{l}\text { Yicld strongth } \\
Y(\text { (iPi) }\end{array}$ & $\begin{array}{l}\text { Hardiless } \\
H\left(\text { CiP }_{i 1}\right)\end{array}$ \\
\hline TiN Sample \#I & $\$ 27$ & 305 & 0.25 & 1.3 .5 & 27 \\
\hline TiN Siample \#? & 4.50 & 301 & 0.25 & 14.5 & 27 \\
\hline HSS & 218 & 218 & 0.30 & 1.8 & 5 \\
\hline Diamsund & $\mid 1+1$ & & 0.07 & 3.5 .7 & yx \\
\hline
\end{tabular}

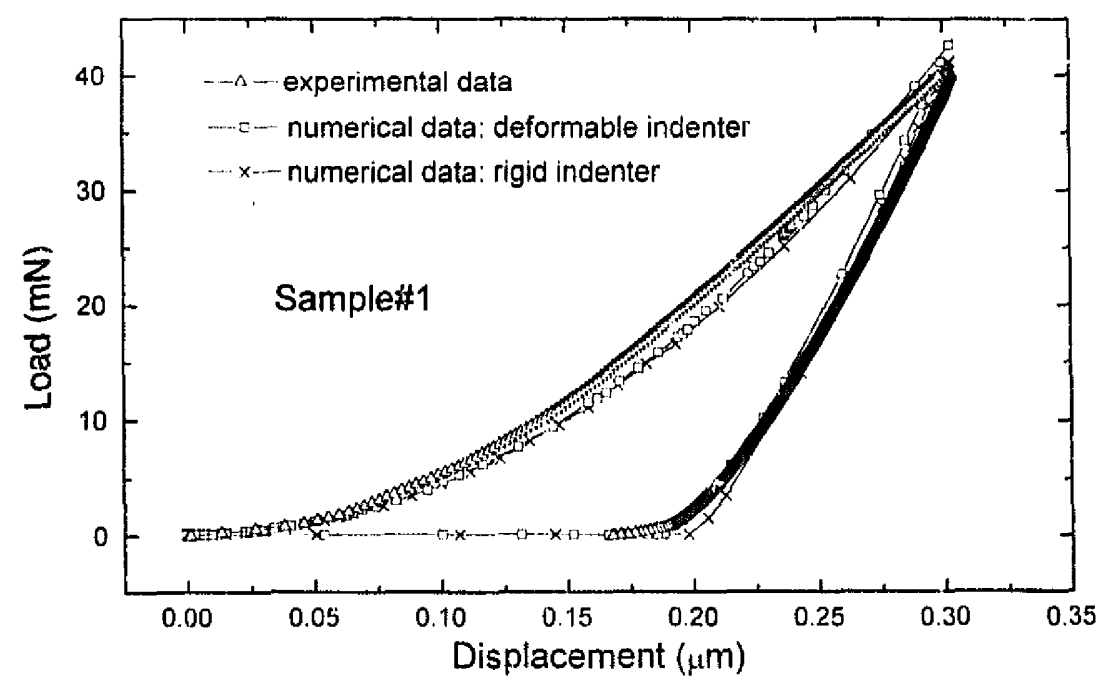

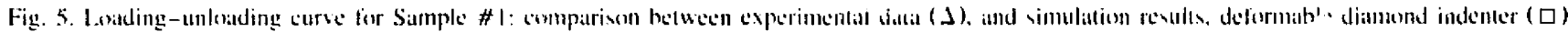
and rigid indenter $(x)$.

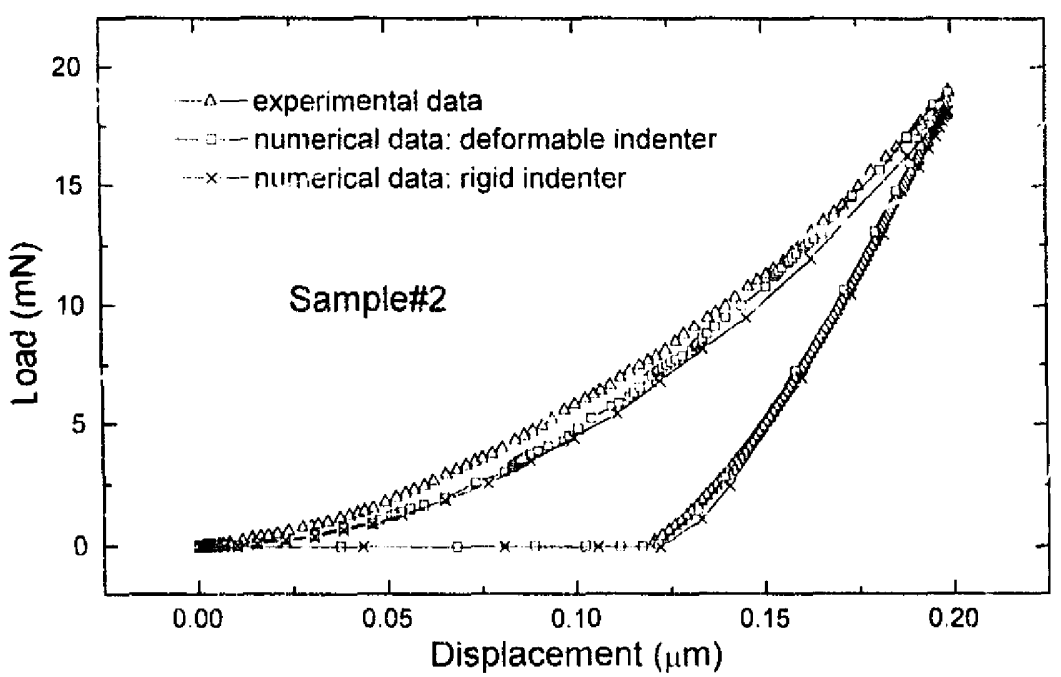

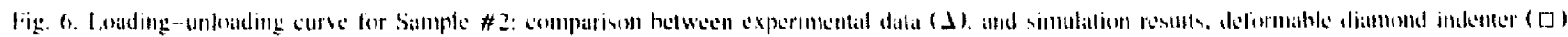
and rigid indentur $(x)$. 


\section{Results and discussion}

\section{4.l. Comparisom with experimental data}

The nanoindentation experiments have been performed on two magnetron sputtered fïms grown with different deposition parameters. Two coatings with a thickness of $2.6 \mu \mathrm{m}$ (Sample \#1) and 3.0 $\mu \mathrm{m}$ (Sample \#2) have been studied by nanoindentation using penetration depths of 0.3 $\mu \mathrm{m}$ and $0.2 \mu \mathrm{m}$ respectively. i.e. $12 \%$ and $6 \%$ of the film thickness.

The Young's modulus $E$ of the coating introduced in the analysis is determined from Eq. (1). It depends on the stiffness of the tip since $E_{\mathrm{r}}$ is evaluated from the nanoindentation experiment. In the numerical analysis. we use either a rigid surface or a deformable tip as an indenter model. For a rigid indenter, $E, \rightarrow \infty$ and the last term of Eq. (1) can be neglected, whereas for a deformable indenter $E_{\text {, }}$ has been taken to be equal to the elastic modulus of diamond. The imput values of $E$ for the FEM analyses are reported in Table 1.

The values of yield strength $Y$ found in literature for TiN [4.6] were too low for the perfect elastic-plastic model that we have considered. In tact, loading-unloading curves obtained using those values do not faithfully reproduce the behavior of the experimental data. In particular, the maximum load is lower. We have tried to improve the agreement by increasing the yicld strength of the film. The best value has been found to be $=14$ GPa for both samples as shown in Table 1. This result is consistent with the linear dependence of $H / Y$ versus $E / Y$ as reported by Tabor [12]. For matcrials having a ratio $l: Y$ of the oncter of 10. $H / Y=1.5$ and for $E / Y$ greater than $100, H$ is about $3 Y$. In our case $E / Y \simeq 32$ and the ratio $H / Y=2$ gives a value lying on the straight line within the indicalted limits,

In Figs. 5 and 6 , the experimental data and the ntmeri- cal results are shown for Sample \#1 and Si rple \#2 respectively.

The agreement between ihe experimental results and the finite element calculations is satisfactory. in particular in the first part of the unloading curve where the Young : modulus is evaluated.

The loading curves show a larger deviation than the unloading curves. This may be due to differences in the actual and assumed yield strength or due to the use of a constitutive model of materials like the perfect elastic-plastic one without work hardening rate. Other causes of the disagreement could be the rounding of the tip as a result of wear [13].

The comparison between the results for the two different models of the tip shows no relevant difierences. The only significant discrepancy is the value of the maximum load. which is slightly higher in the case of the deformable tip. This is due to the partial absorption of the deformation ancrgy from the indenter.

The three-dimensional model with perfectly sharp Berkovich indenter has been used for testing the validity of the axisymmetric model. In particular. we want to verify if the use of a pyramidal indenter could better reproduce the experimental loading curve. As suggested by the axisymmetric analysis, no relevant differences are apparent between the two models of the indenter. Therefore, we have chosen to model the tip as a rigid surface in order to restrain the degrees of ireedom.

The loading-unloading curve of Sample \# I has been simulated with the three-dimensional model. In Fig. 7 the results of the analysis are compared with the corresponding responding data of the axisymmetric model. The curves are practically identical indicating that the axisymmetric model with a conical indenter. which has the same area function of the real tip. is an effective tool for simulating nanoindentation procedure with a Berkovich indenter.

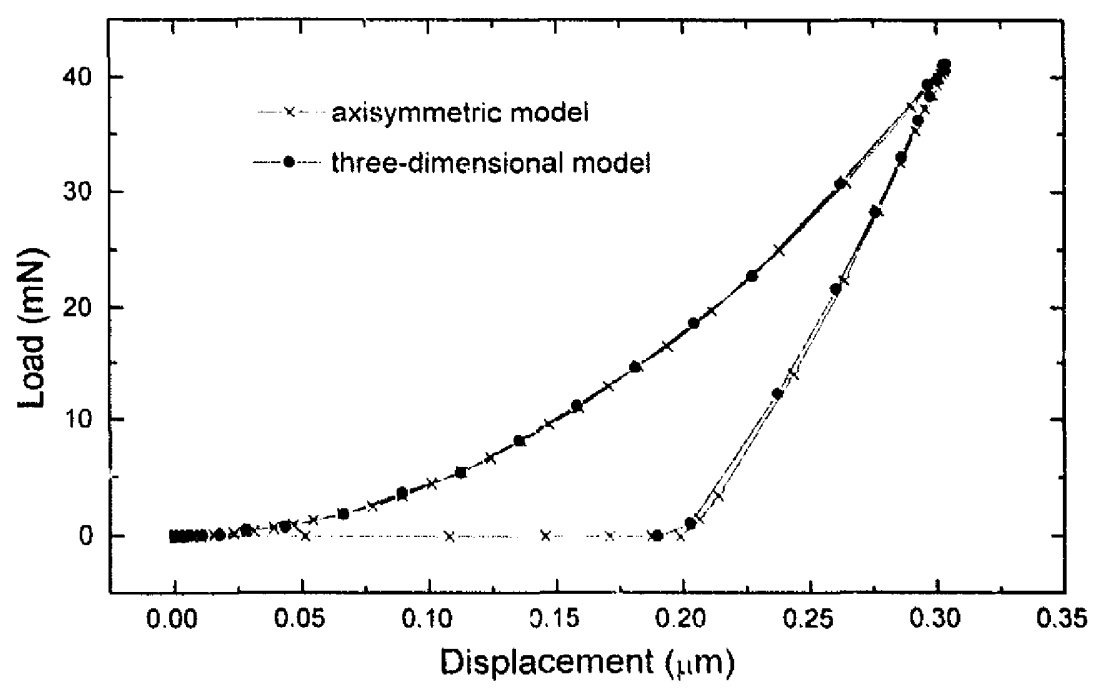

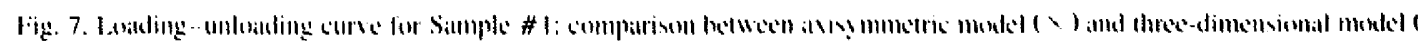




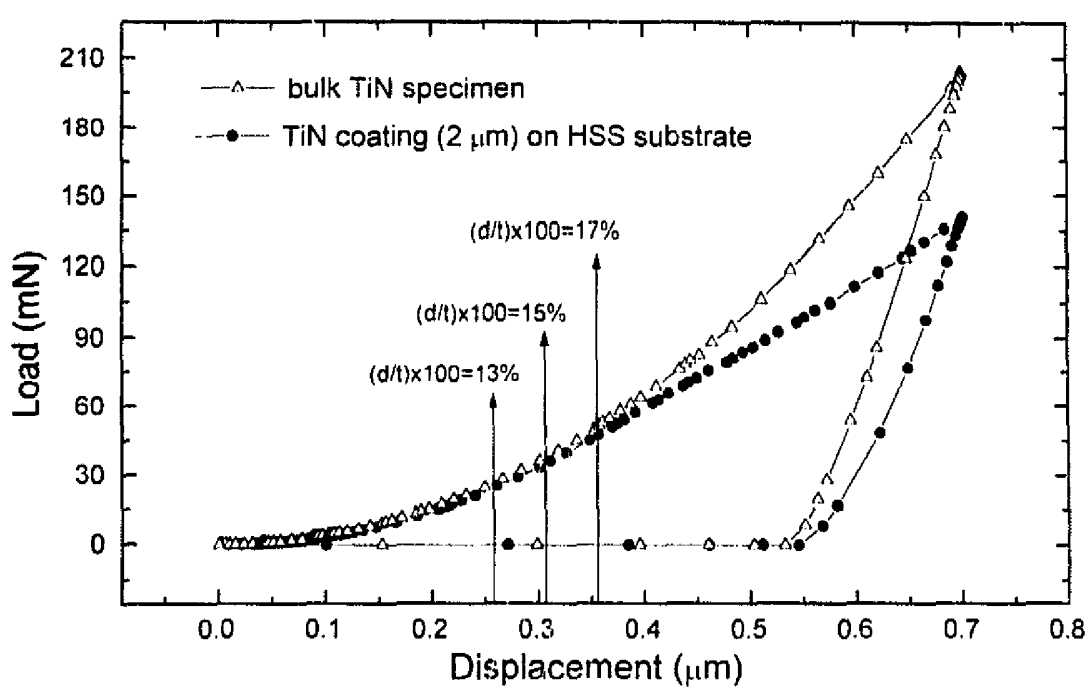

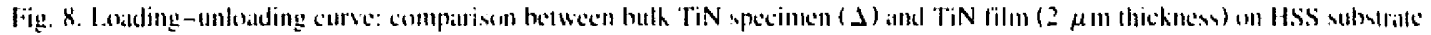
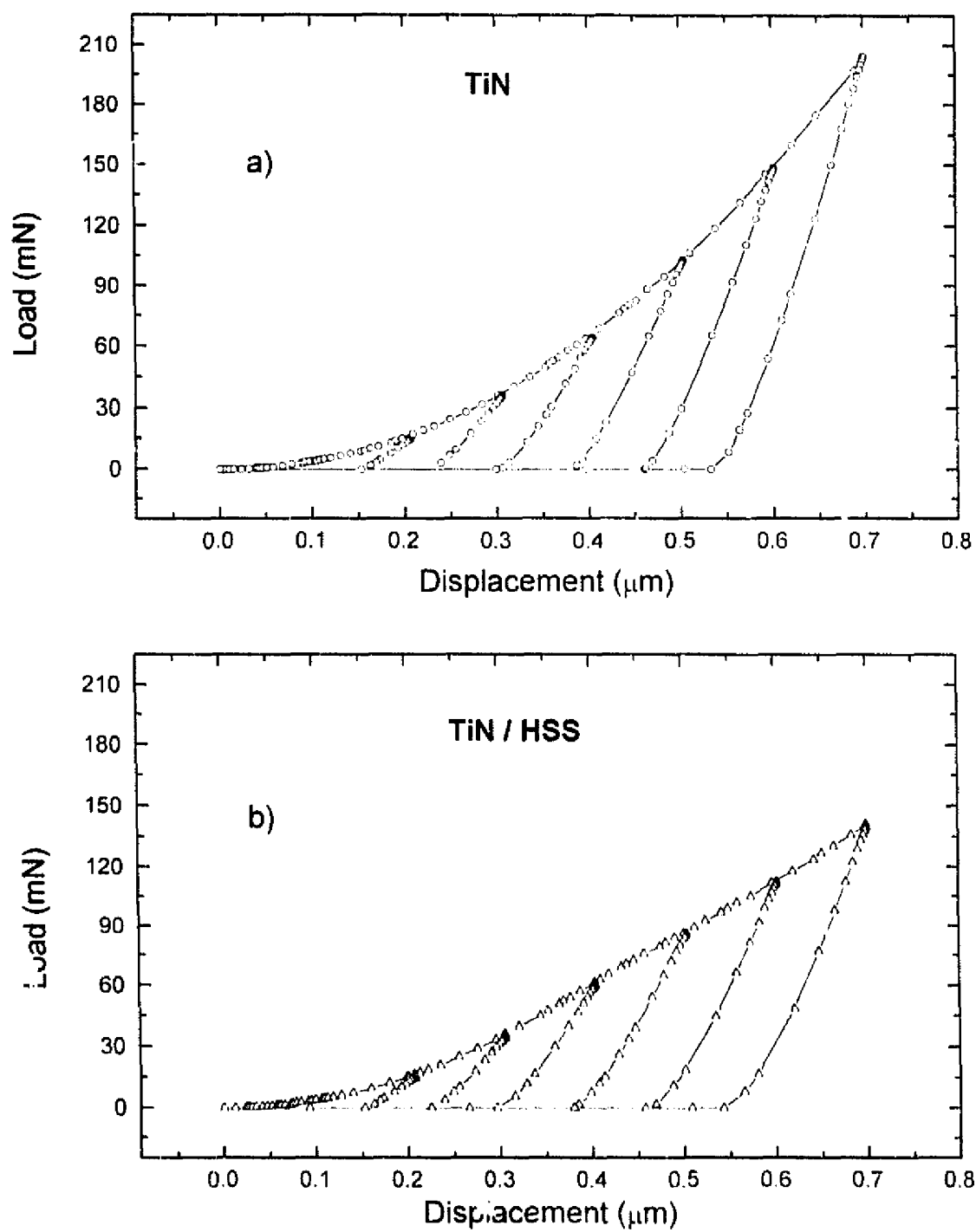

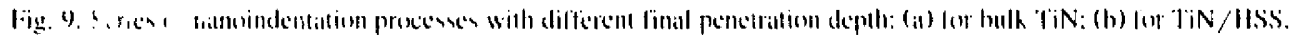




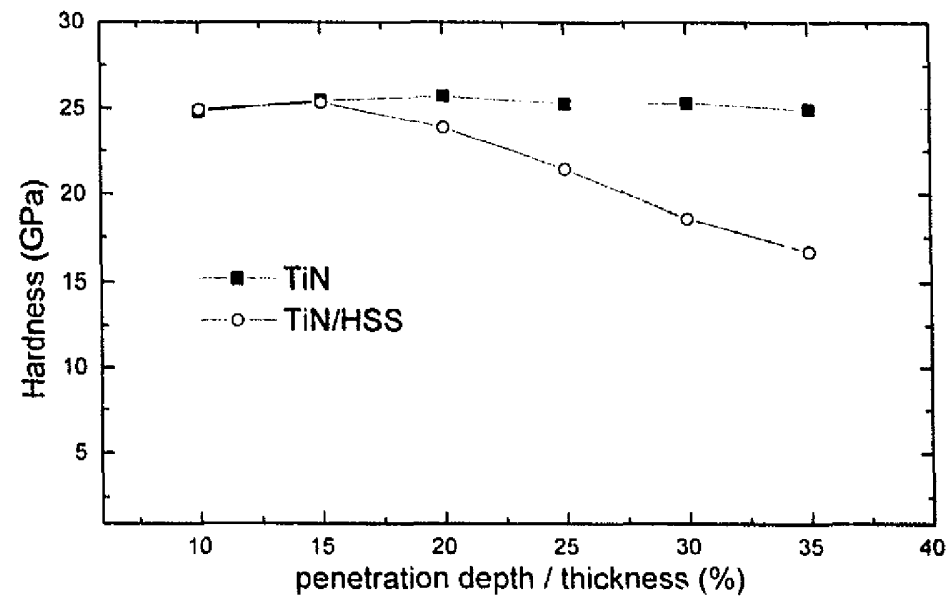

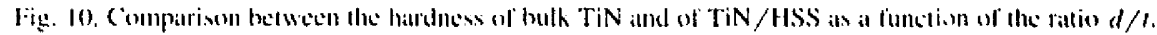

\subsection{Lond ierwas pene'tration depth for conated systems}

We have used the axisymmetric model described previously for studying the very complex stress-strain field induced by the indentation. In particu!ar, we have studied the effect of the substrate on the distribution of the plastic deformation tor coated systems as a function of the penetration depth. In the hardness measurements, the penetration depth is chosen not to exceed 10-20\% of the film thickness in order to avoid substrate effects. In our simulation. we have compared a bulk TiN specimen with a $2 \mu \mathrm{m}$ thick TiN coating on HSS substrate The indenter penetrated up to $0.7 \mu \mathrm{m}$. corresponding to $35 \%$ of the film thickness.

Fig. 8 shows the results of the numerical analysis for the bulk TiN (open triangles) and for the TiN/HSS system (solid circles). At a value of penetration d equal to $1.5 \%$ of the film thickness t, the two loading curves begin to diverge. The curve of the coaled specimen changes its curvature and the final penctration is reached for a $30 \%$ smaller load than in the case of the bulk material. There- fore, the hardness evaluation is strongly affected by the ratio $d / l$. For a quantitative evaluation of the error on the hardness measurements due to the substrate stiffness, we have performed a series of numerical analyses for both the bulk TiN and the TiN/HSS system with different final penetration. In Fig. 9a and $b$ the load versus depth for the two cases are shown. The hardness. as a function of the ratio $d / t$, is drawn in Fig. 10, For the coated specimen. from $d / t \times 100=15 \%$ to $d / t \times 100=35 \%$. the hardness of TiN/HSS svetem with respect to bulk TiN decreases. up to a maximum deviation of $33 \%$.

The analysis of the plastic field during the nanoindentalion process indicates a correlation hetween the onset of the substrate plastic deformation at the interface and the initial separation of the loading curves. Fig. II shows the history of the plastic deformation of the costed specimen. In order to be able to detect the beginning of the first relevant plastic deformation in the substrate. we have chosen to refine the interval of the equivalent plastic strain variable under a threshold value of 4.E-2. In Fig. I Ia. the penetration depth is $13 \%$ of the film thickness. At this
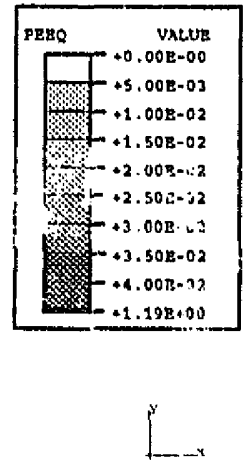

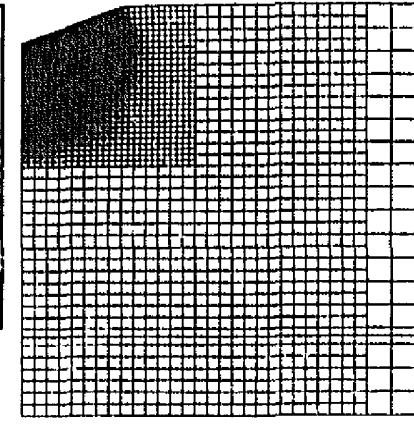

c)

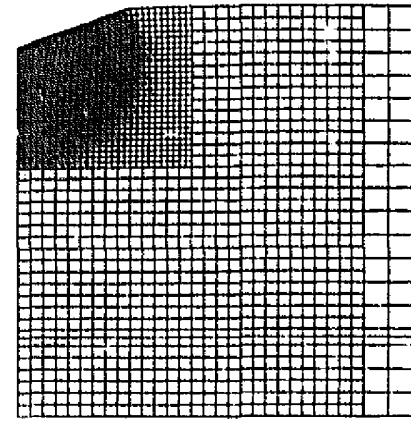

b)

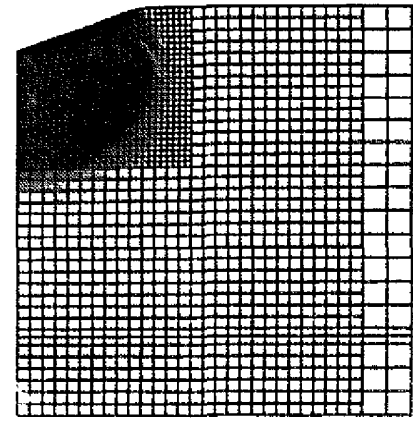

c)

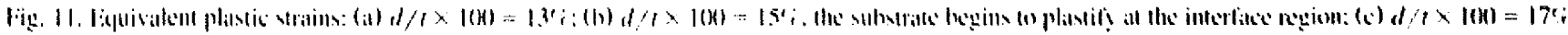

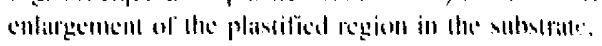


stage of the indentation. only the film is plastically deforried. At $d / t=0.15$, the substrate shows plastic deformation at the interface whereas the coating does not (see Fig. I $(b)$. This is exactly the $d / l$ value at which the loading curve of the bulk TiN deviates from that of the TiN coating. Fig. $1 / \mathrm{c}$ ) shows an even larger plastic region for $d / t=0.17$. The three $d / t$ values are indicated in Fig. 8.

\section{Conclusions}

In this work we have studied the Berkovich nanoindentation process by means of finite element analysis. We have verified that an axisymmetric model gives the same results as a three-dimensional model if the conical indenter has the same area function as a Berkovich lip. Since the axisymmetric model has a lower number of degrees of freedom and, consequently. requires shorter computational time. it was used for all the anaiyses presented. For testing the feasibility of the model we compared experimentil data of a nanoindentation measurement performed on a TiN/HSS specimen with numerical results. The loadingunloading curves present good agreement. even if there is a slight discrepancy in the loading section.

The effect of the substrate stilifiess on conting hardne'ss measurements wats analyzed for the TiN/HSS system through the evolution of the piastic deformation field. The initiation of plastic deformation in the substrate at the interface zone corresponds to a penetration depth of the indenter equal to $15 \%$ of the film thickness. This relative penetration of the indenter represents a critical point from which the load data begin to be affected by the lower stiffiness of the subsarate. Consequently. at this stage the hardness of the film decreases with respect to the real value. Thus. the general criterion that the film should not be penetrated more than 10-20\% of its thickness is still acceptable for TiN on HSS.

\section{Acknowledgements}

The authors wish to thank W. Gissler and H. Stamm for helpful discussions. A. Holfmamn and T. Sasaki for valuable technical assistance. One of the authors is grateful to the Laboratorio Materiali Innovativi (LaMl) of the University of Basilicata (Italy) and to the Regiome Batsilicala for their financial support.

\section{References}

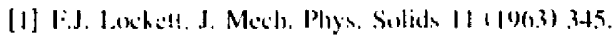

[2] 1). Tilbur. Rev, liby, Tech, I (19)7(1) 1-15.

[3] K.I. Johntinon, J. Mech. Phys, Solids I8 (1970) I15.

[.t] X. Ciri. H. Bangert. Thin Solid Filns $26+(1045)$ 5!?

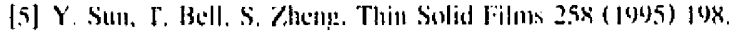

[0] H.I: Wallg. H. Ballgert. Mater. Sic. Eng, $\Lambda l 6(\mid 0), 3), 34,3$.

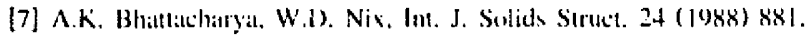

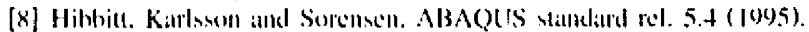

[i] W.C. Oliver. (i.M. Pharr. J. Malter. Res, 7 (140)2) 1.564.

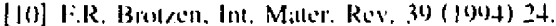

[11] IN. Sneddm, Int. J. Ling S.i. 3 (196,5) 47.

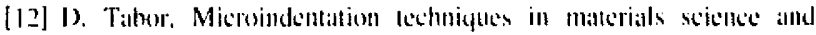
engimering. ASTM. STP'B80 (1984).

[1.3] C.W. Shih. M. Yang. J.C.M. I.i. I. Matter. Kes, 6 (|4)|) 2027 . 